07,01

\title{
Анализ размерных эффектов при мартенситных переходах в эпитаксиальных пленках и микрочастицах сплава $\mathrm{Ni}-\mathrm{Mn}-\mathrm{Sn}$
}

\author{
(C) Г.А. Малыгин \\ Физико-технический институт им. А.Ф. Иофрфе РАН, \\ Санкт-Петербург, Россия \\ E-mail: malygin.ga@mail.ioffe.ru
}

Поступила в Редакцию 5 марта 2019 г.

В окончательной редакции 5 марта 2019 г.

Принята к публикации 5 марта 2019 г.

В рамках теории размытых мартенситных переходов (РМП), основанной на термодинамических и кинетических соотношениях, анализируются имеющиеся в литературе данные по влиянию толщины эпитаксиальных пленок (films) и размера кристаллитов в микрочастицах порошка сплава $\mathrm{Ni}-\mathrm{Mn}-\mathrm{Sn}$ на параметры мартенситных переходов в этом сплаве. Целью анализа является установление явной (функциональной) зависимости этих параметров от толщины пленки $D$ и размера нанокристаллитов $d$ в пленке и микрочастицах. Результаты дифракционного R-анализа показывают, что из-за когерентной связи эпитаксиальной пленки с твердой подложкой или в результате интенсивной пластической деформации сплава при его размоле в шаровой мельнице в пленках и микрочастицах порошка сплава возникают внутренние упругие микродеформации и напряжения. Анализ показывает, что локальные микронапряжения существенно влияют на вид зависимости температурного интервала (размытия) перехода $\Delta T$ от размерных факторов $D$ или $d$. В отсутствие микронапряжений эти зависимости имеют вид $\Delta T \sim 1 / D^{2}$ или $\Delta T \sim 1 / d^{2}$. При наличии микронапряжений температурный интервал перехода зависит от размерных факторов, как $\Delta T \sim 1 / D$ или $\Delta T \sim 1 / d$.

Ключевые слова: сплавы ЭПФ, размерные эффекты, эпитаксиальные пленки, мартенситные переходы.

DOI: 10.21883/FTT.2019.07.47843.406

\section{1. Введение}

Ферромагнитные сплавы $\mathrm{Ni}-\mathrm{Mn}-\mathrm{Ga}$ с эффектом температурной и магнитной памяти формы с замещением Ga на атомы $\mathrm{Sn}[1-5]$, In [6] или $\mathrm{Sb}$ [7] привлекли в последнее десятилетие внимание исследователей как обладающие более широким спектром функциональных и технологических свойств по сравнению с известным сплавом $\mathrm{Ni}_{2} \mathrm{MnGa}$. Указанные сплавы имеют более высокую чувствительность к изменению температуры и магнитного поля и более значительную величину механокалорического и магнетокалорического эффектов $[1,8]$. В связи с миниатюризацией различных робототехнических, сенсорных и криогенных устройств особое внимание уделяется в настоящее время исследованию влияния размеров $D$ монокристальных образцов сплавов, а в нанокристаллических образцах - размеров зерен $d$, на мартенситные переходы в этих сплавах и, следовательно, влияния размерных факторов на функциональные свойства сплавов в диапазоне размеров $d, D=10-100 \mathrm{~nm}$.

Так, резистометрическое исследование прямого и обратного мартенситных переходов в эпитаксиальных пленках сплава $\mathrm{Ni}_{51.6} \mathrm{Mn}_{32.9} \mathrm{Sn}_{15.5}$ толщиной 20-200 nm, приготовленных на подложках из монокристаллов $\mathrm{MgO}$, показало [2], что ширина перехода по температуре $\Delta T$ возрастает с уменьшением толщины пленки. Найдено также, что температуры равновесия мартенситной и аустенитной фаз при прямом, $T_{c}^{M}=\left(M_{s}+A_{f}\right) / 2$, и обратном, $T_{c}^{A}=\left(A_{s}+M_{f}\right) / 2$, переходах резко снижаются и обращаются в нуль при критической толщине пленки $D_{k} \approx 20 \mathrm{~nm}$. При меньших толщинах мартенситный переход в пленке отсутствует, она остается в аустенитном состоянии. Наблюдаемые размерные эффекты авторы связывают с влиянием на параметры мартенситных переходов нескольких факторов, а именно, наличия подложки и связанной с этим разницы (mismatch) постоянных решеток аустенита и подложки, деформационной совместимости (compatibility) мартенситной и аустенитной фаз и с влиянием собственно размерного фактора.

В [3] при исследовании мартенситного перехода в эпитаксиальных пленках сплава $\mathrm{Ni}_{50} \mathrm{Mn}_{35} \mathrm{Sn}_{15}$ толщиной от 120 до $2500 \mathrm{~nm}$, изготовленных на $\mathrm{Si}$-подложках, был получен парадоксальный результат, а именно, отсутствие в пленках толщиной $120 \mathrm{~nm}$ и меньше мартенситного перехода, но переход имел место при толщине пленки $400 \mathrm{~nm}$ и выше. Этот результат находится в противоречии с данными [2], согласно которым стабилизация аустенита в пленках сплава $\mathrm{Ni}-\mathrm{Mn}-\mathrm{Sn}$ по составу близкого к [3] наступает при толщине пленок меньше $20 \mathrm{~nm}$.

Еще в одном исследовании сплав $\mathrm{Ni}-\mathrm{Mn}-\mathrm{Sn}$ аналогичный по составу сплаву [3] подвергался размолу в шаровой мельнице $[4,5]$. Полученный порошок отжигался в течение 5 min при четырех температурах в интервале $573-873 \mathrm{~K}$, и исследовался с помощью DS-калориметра, рентгеновской дифракции [4] и мессбаурерской спектроскопии [5] на предмет влияния отжига на параметры перехода микрочастиц порошка из аусте- 
нитного в мартенситное состояние. В результате было установлено, что температурная ширина перехода $\Delta T$ и ширина дифракционных пиков (величина внутренних упругих деформаций $\varepsilon$ в частицах порошка) уменьшаются с ростом температуры отжига. Наблюдаемые эффекты авторы связывают с влиянием деформационных дефектов (упругих полей дислокаций) на параметры мартенситных переходов в микрочастицах сплава.

В рассмотренных выше работах имеется лишь качественный анализ полученных результатов, поскольку в настоящее время отсутствует чувствительная к структуре сплава на мезоуровне теория обратимых термоупругих мартенситных переходов в сплавах с эффектом памяти формы (ЭПФ), каким является сплав $\mathrm{Ni}-\mathrm{Mn}-\mathrm{Sn}$. Наиболее близкой к такой теории является в настоящее время теория размытых мартенситных переходов (РМП) [9,10]. Она базируется как на термодинамических, так и кинетических уравнениях и соотношениях, что позволяет учитывать на мезоуровне влияние размерных факторов на кинетику и параметры мартенситных переходов, а, следовательно, и на функциональные свойства различных устройств, использующих сплавы с ЭПФ в качестве рабочих элементов.

Недавно теория РМП была применена в [10] для анализа влияния размерного фактора на параметры мартенситного перехода в сплаве TiNi в виде наночастиц сферической формы диаметром $D=3-24 \mathrm{~nm}$ [11]. А также для анализа влияния этого фактора на кривые сжатия микрокристаллов сплава $\mathrm{Ni}-\mathrm{Fe}-\mathrm{Ga}$ в направлении оси [011] в виде столбиков (pillars) диаметром от 0.235 до $10 \mu \mathrm{m}$ [12]. Было установлено [10], что существуют два источника размерных эффектов в этих кристаллах: термодинамический, который определяет температурную ширину (размытие) мартенситного перехода, как $\Delta T \sim 1 / D^{2}$, и кинетический, определяющий существование критического поперечного размера кристалла $D_{k}$; при меньших сечениях кристалл остается в аустенитном состоянии. Целью настоящей работы является анализ в рамках теории РМП влияния толщины эпитаксиальных пленок $D$ [3] и размеров кристаллитов (нанозерен) $d$ в пленках [3] и микрочастицах порошка [4] сплава $\mathrm{Ni}-\mathrm{Mn}-\mathrm{Sn}$ на параметры мартенситных переходов в этом сплаве. Анализ показывает, что ширина фазового перехода (его размытие по температуре) изменяется с толщиной пленки и размером кристаллитов $d$, соответственно, как $\Delta T \sim 1 / D$ и $\Delta T \sim 1 / d$. Установлены также явные зависимости других параметров мартенситных переходов в этих сплавах от размерного фактора. В результате анализа находит также объяснение парадоксальный характер полученного в [3] результата, а именно, отсутствие мартенситного перехода в пленках сплава $\mathrm{Ni}-\mathrm{Mn}-\mathrm{Sn}$ при их толщине $120 \mathrm{~nm}$.

\section{2. Основные соотношения теории РМП}

Как сказано выше, теория РМП базируется как на термодинамических, так и на кинетических соотношениях.
Основное термодинамическое соотношение в случае одностадийного мартенситного перехода имеет вид $[9,10]$

$$
\varphi_{M}=\frac{1}{1+\exp (\Delta U / k T)}, \quad \varphi_{A}=1-\varphi_{M},
$$

где $\varphi_{M}$ и $\varphi_{A}$ - относительные объемные доли мартенсита и аустенита в сплаве, $\Delta U=\omega(D, d) \Delta u$ - изменение свободной энергии сплава при образовании в нем элементарного объема новой фазы $\omega_{D, d}$, ограниченного (стесненного, constrained) размерами $D$ или $d$,

$$
\frac{1}{\omega(D, d)}=\frac{1}{\omega_{0}}+\frac{1}{\omega_{D, d}}
$$

$\omega_{0}$ - элементарный объем в отсутствие пространственного стеснения превращения,

$$
\Delta u=q \frac{T-T_{c}}{T_{c}}-\varepsilon_{m} \sigma,
$$

- объемная плотность свободной энергии мартенситного перехода, $q$ - теплота перехода, $T-$ температура, $T_{c}$ - температура объемного равновесия мартенситной и аустенитной фаз, когда $\varphi_{M}=\varphi_{A}=1 / 2, \varepsilon_{m}$ - деформация превращения, $\sigma$ - механическое напряжение, приложенное к кристаллу, $k$ - постоянная Больцмана.

Кинетику фазового перехода определяют кинетические уравнения для объемных долей мартенситной $\varphi_{M}$ и аустенитной $\varphi_{A}$ фаз $[9,10]$. Уравнения описывают распределение мартенсита и аустенита в кристалле при прямом и обратном мартенситных переходах. Неоднородное распределение фаз является результатом процесса самоорганизации дислокаций превращения, параметры его зависят от температуры, напряжения, структурных и размерных факторов. В [10] приведено частное решение этих уравнений в случае равенства объемных долей мартенсита и аустенита (равенства размеров мартенситных и аустенитных ламелей) в кристалле сплава. Оно позволяет найти зависимость температуры равновесия фаз при прямом $T_{c}^{M}$ и обратном $T_{c}^{A}$ мартенситных переходах от размерного фактора,

$$
\begin{aligned}
T_{c}^{M} & =\left[1+\frac{k T_{c 0}}{q \omega(D, d)} \ln \left[\frac{3}{2} k_{0}(D, d)-1\right]\right] T_{c 0}, \\
T_{c}^{A} & =\left[1+\frac{k T_{c 0}}{q \omega(D, d)} \ln \left[3 k_{0}(D, d)-1\right]\right] T_{c 0} .
\end{aligned}
$$

а также разницу между ними $\Delta T=T_{c}^{A}-T_{c}^{M}$, определяющую ширину (размытие) перехода по температуре и ее зависимость от размерного фактора (в рассматриваемом случае - толщины пленки $D$ или размера кристаллитов в ней $d$ ),

$$
\Delta T_{A M}=\left[\frac{k T_{c 0}}{q \omega(D, d)} \ln \left(\frac{3 k_{0}(D, d)-1}{3 k_{0}(D, d) / 2-1}\right)\right] T_{c 0} .
$$

где $T_{c 0}-$ температура равновесия фаз в отсутствие стеснения переходов,

$$
k_{0}(D, d)=\left(\frac{D, d}{\pi^{1 / 2} D_{c}}\right)^{2},
$$

- критический размер петель при гомогенном зарождении дислокаций фазового превращения. Соотноше- 
ние (3b) определяет вклад кинетического фактора в размерный аспект фазового превращения.

\section{3. Сравнение с экспериментом}

\section{1. Эпитаксиальные пленки}

Рис. 1 демонстрирует зависимость температур равновесия фаз в пленках сплава $\mathrm{Ni}_{51.6} \mathrm{Mn}_{32.9} \mathrm{Sn}_{15.5}$ [2] при прямом $T_{c}^{M}=\left(M_{s}+A_{f}\right) / 2$ и обратном, $T_{c}^{A}=\left(M_{f}+A_{s}\right) / 2$, мартенситных переходах от толщины пленки $D$, где $M_{s}, M_{f}$ и $A_{s}, A_{f}$ - температуры начала и конца, соответственно, прямого и обратного мартенситных переходов (серия экспериментов $A[2]$ ). Видно, что температура $T_{c}^{M}$ при прямом мартенситном переходе начинает резко снижаться при толщине пленки меньше $30 \mathrm{~nm}$ и обращается в нуль при критической ее толщине $D_{k} \approx 20 \mathrm{~nm}$. При обратном мартенситном переходе снижение температуры $T_{c}^{A}$ по мере уменьшения толщины пленки происходит более плавно и до более низких значений, и заканчивается ее обращением в нуль примерно при той же критической толщине пленки, что и при прямом переходе. Согласно соотношениям (3) зависимость температур объемного равновесия фаз от толщины пленки $D$ определяется зависимостью от нее элементарного объема фазового превращения $\omega(D)(1 \mathrm{~b})$ и кинетического фактора $k_{0}(D)(3 \mathrm{~b})$. Рассмотрим в начале зависимость элементарного объема превращения от $D$. Здесь могут иметь место две ситуации. Первая стеснение фазового превращения не сопровождается возникновением упругих деформаций. Такая ситуация

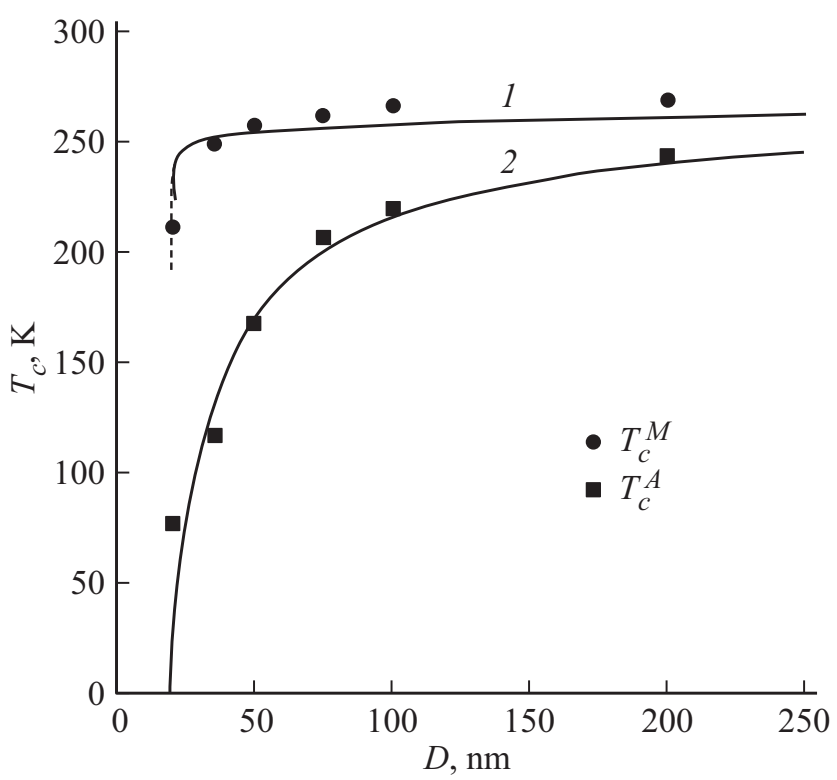

Pис. 1. Зависимость температур равной объемной концентрации мартенсита и аустенита при прямом $T_{c}^{M}$ и обратном $T_{c}^{A}$ мартенситных переходах в эпитаксиальных пленках сплава $\mathrm{Ni}_{51.6} \mathrm{Mn}_{32.9} \mathrm{Sn}_{15.5}$ от толщины пленки $D$ [2]. Кривые 1 и пунктир - расчет согласно уравнениям (2), кривая $2-$ согласно уравнению (8). наблюдается, например, при мартенситном переходе в равновесной, хорошо отожженной нанокристалической структуре сплава TiNi [11]. Как показано в [10,13], элементарный объем превращения определяется в этом случае соотношением $\omega \sim d^{2}$, где $d-$ размер кристаллитов. Вторая ситуация возникает, когда стеснение мартенситного превращения сопровождается образованием упругих микродеформаций, как это имеет место, например, в сплаве $\mathrm{Cu}-\mathrm{Al}-\mathrm{Ni}$, содержащем когерентные с матрицей частицы двуокиси гафния с объемной концентрацией $1 \%$. В этом случае элементарный объем превращения зависит от поперечного размера частиц $d$, как $\omega \sim d$ [14]. В пленках сплава $\mathrm{Ni}-\mathrm{Mn}-\mathrm{Sn}[2]$ элементарный объем превращения ограничен толщиной пленки $D$ и образованием петли дислокаций превращения площадью $D^{2}$. Следовательно, для элементарного объема фазового превращения имеем соотношение

$$
\omega_{D}(D)=a_{0} D^{2}
$$

где $a_{0}=a / \sqrt{2}-$ расстояние между габитусными плоскостями кристалла, $a-$ постоянная решетки аустенита.

Рассмотрим теперь влияние кинетического факторана $k_{0}(D)$ на зависимости температур равной объемности фаз $T_{c}$. Согласно формулам (2) и приведенным на рис. 1 результатам [2] температуры равного объемного содержания мартенсита и аустенита в пленке обращаются в нуль при величине кинетического фактора $k_{0}(D)=2 / 3$ и $k_{0}(D)=1 / 3$ и $D=D_{k} \approx 20 \mathrm{~nm}$, соответственно, при прямом и обратном мартенситных переходах. Эти соотношения позволяют оценить величину критического размера петель $D_{c}^{M} \approx 13.8 \mathrm{~nm}$ и $D_{c}^{A} \approx 19.5 \mathrm{~nm}$. Сравним элементарный объем превращения (4) и объем превращения в отсутствие стеснения мартенситного перехода $\omega_{0}=\left(\pi D_{0}^{2} / 4\right) a_{0}$, где $D_{0}=D_{c}$. Для этого формулу $(1 \mathrm{~b})$ запишем в виде

$$
\frac{\omega_{0}}{\omega(D)}=1+\frac{\omega_{0}}{\omega_{D}}, \quad \frac{\omega_{0}}{\omega_{D}}=\frac{\pi}{4}\left(\frac{D_{c}}{D}\right)^{2} .
$$

Подставляя в правую часть второго соотношения (5) $D_{c}=14-19 \mathrm{~nm}$ и наименьшее значение толщины пленки $D=D_{k}=20 \mathrm{~nm}$, когда в пленке не образуется мартенсит, получаем оценку величины отношения $\omega_{0} / \omega_{D} \approx 0.4-0.7$. Согласно этой оценке стеснение элементарного объема превращения вследствие его ограничения толщиной пленки не должно существенно влиять на температуры $T_{c}^{M}$ и $T_{c}^{A}$ (рис. 1). Следовательно, их зависимость от толщины пленки определяется, в основном, кинетическим фактором $k_{0}(D)$. На рис. 1 кривая 1 и пунктир проведены согласно уравнениям (2) и (6) при $k T_{c 0} / q \omega_{0}=10^{-2}$ и $T_{c 0}=250 \mathrm{~K}$. Видно, что в случае прямого мартенситного перехода (кривая 1 ) теория согласуется с экспериментом, а при обратном переходе между теорией (пунктир) и экспериментом имеется существенное расхождение.

В пленках сплава $\mathrm{Ni}-\mathrm{Mn}-\mathrm{Sn}$ [2], выращенных на подложках из кристаллов $\mathrm{MgO}$, рентгеновская дифракция показывают наличие в пленках упругих микродеформа- 

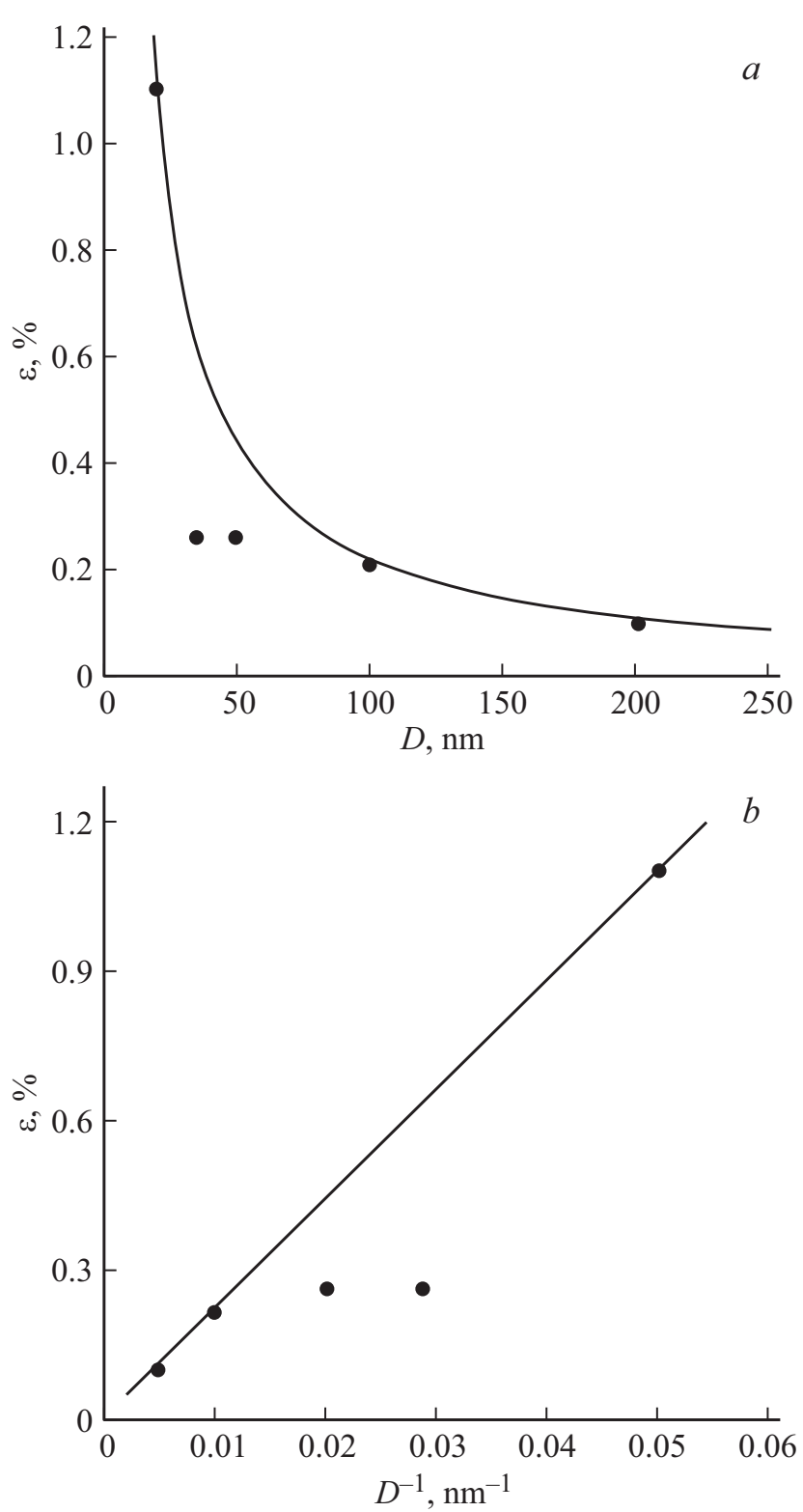

Рис. 2. Зависимость упругих микродеформаций в эпитаксиальных пленках сплава $\mathrm{Ni}_{51.6} \mathrm{Mn}_{32.9} \mathrm{Sn}_{15.5}$ от толщины пленки в прямых $(a)$ и обратных $(b)$ координатах [2]. Кривые - расчет по формуле (6).

ций $\varepsilon$, зависящих от толщины пленки. На рис. $2, a$ и $2, b$ показана эта зависимость в прямых, $\varepsilon-D$, и обратных, $\varepsilon-1 / D$, координатах. Видно, что величина деформации снижается с ростом толщины пленки. Зависимость $\varepsilon(D)$ может быть аппроксимирована формулой

$$
\varepsilon(D)=\delta_{0} \frac{D_{k}}{D}
$$

где $\delta_{0}$ - относительная разница (mismatch) параметров решетки подложки и аустенита сплава при $D=D_{k}$. На рис. 2 кривые $\varepsilon(D)$ демонстрирует эту зависимость при $\delta_{0}=1.1 \cdot 10^{-2}$ и $D_{k}=20 \mathrm{~nm}$. Что касается отмеченного выше расхождения между теорией и экспериментом при обратном мартенситном превращении, то можно предположить, что оно вызвано напряжениями $\sigma_{e}(D)=\mu_{A} \varepsilon(D)$ из-за когерентного характера связи пленки с подложкой

$$
\sigma_{e}(D)=\delta_{0} \mu_{A} \frac{D_{k}}{D},
$$

где $\mu_{A}-$ модуль сдвига аустенита. Подставляя напряжение (7) в (1c), получим вместо (2b) и (3a) следующие соотношения

$$
\begin{aligned}
& T_{c}^{A, \sigma}(D)=\left[1-\delta_{\sigma} \frac{D_{k}}{D}+\frac{k T_{c 0}}{q \omega(D)} \ln \left[3 k_{0}(D)-1\right]\right] T_{c 0}, \\
& \Delta T_{\sigma}(D)=\left[\delta_{\sigma} \frac{D_{k}}{D}+\frac{k T_{c 0}}{q \omega(D)} \ln \left(\frac{3 k_{0}(D)-1}{3 k_{0}(D) / 2-1}\right)\right] T_{c 0},
\end{aligned}
$$

где $\delta_{\sigma}=\delta_{0} \varepsilon_{m} \mu_{A} / q$. На рис. 1 кривая 2 построена согласно соотношению (8) при $D_{k}=20 \mathrm{~nm}$ и величине параметра $\delta_{\sigma}=0.8$. Видно, что учет микронапряжений позволяет согласовать теорию и эксперимент. Другая возможная причина рассматриваемого расхождения между теорией и экспериментом - это поверхностная энергия пленки $[10,15]$. Согласно $[10,15]$ снижение температуры равновесия фаз из-за поверхностного натяжения определяется уравнением $\Delta T_{s}=\left(2 \gamma_{s} / q D\right) T_{c 0}$, где $\gamma_{s}$ - поверхностная энергия. В [10] найдено, что температура $T_{c}^{M}$ в нанокристаллах сплава $\mathrm{TiNi}$ [16] становится чувствительной к поверхностному натяжению при размерах поперечного сечения кристаллов меньше $10 \mathrm{~nm}$. В рассматриваемом случае пленок сплава $\mathrm{Ni}-\mathrm{Mn}-\mathrm{Sn}$ отклонение температуры $T_{c}^{A}$ от соотношения (2b) наступает при гораздо большей величине толщины пленки (рис. 1), т.е. оно, скорее всего, вызвано напряжениями (7).

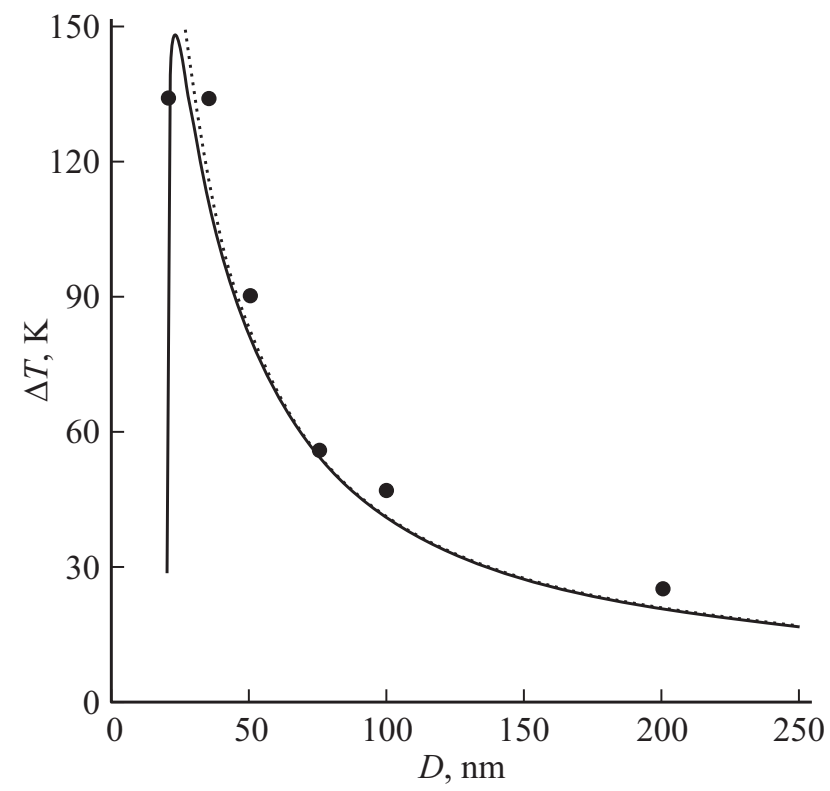

Рис. 3. Зависимость температурной ширины мартенситного превращения $\Delta T$ в эпитаксиальных пленках сплава $\mathrm{Ni}_{51.6} \mathrm{Mn}_{32.9} \mathrm{Sn}_{15.5}$ от толщины пленки [2]. Кривая - расчет согласно уравнению (9), пунктир - вклад микронапряжений (7) в эту зависимость. 
На рис. 3 экспериментальные точки демонстрируют разницу температур $\Delta T=T_{c}^{M}-T_{c}^{A}=\left(M_{s}-M_{f}\right) / 2$ $+\left(A_{f}-A_{s}\right) / 2$ при прямом, $T_{c}^{M}=\left(M_{s}+A_{f}\right) / 2$, и обратном, $T_{c}^{A}=\left(M_{f}+A_{s}\right) / 2$, мартенситных переходах. Она определяет температурную ширину перехода в сплаве $\mathrm{Ni}-\mathrm{Mn}-\mathrm{Sn}$ [2]. Кривая на рисунке иллюстрирует разницу температур $\Delta T(D)=T_{c}^{M}(D)-T_{c}^{A, \sigma}(D)$. Видно, что наблюдается хорошее согласие теории с экспериментом. Пунктиром на рис. 3 обозначен вклад микронапряжений $\Delta T^{\sigma}(D)=\delta_{\sigma}\left(D_{k} / D\right) T_{c 0}$ в зависимость $\Delta T(D)$. Этот вклад является определяющим при толщине пленок $D>20 \mathrm{~nm}$. Экспериментальные данные [2] позволяют на основе теории РМП проанализировать также зависимость гистерезиса $\Delta T_{h}$ электросопротивления пленок от их толщины при прямом $\Delta T_{h}^{M}=A_{f}-M_{s}$ и обратном $\Delta T_{h}^{A}=A_{s}-M_{f}$ мартенситных переходах. На рис. 4 показаны эти зависимости, а также зависимость от $D$ усредненной величины гистерезиса $\Delta T_{h}=\left(\Delta T_{h}^{M}+\Delta T_{h}^{A}\right) / 2$. Усредненная величина практически не зависит от толщины пленки и равна $15.5 \mathrm{~K}$, а частные ее значения могут быть аппроксимированы полуэмпирическими формулами

$$
\begin{gathered}
\Delta T_{h}^{M}=15.5+0.03\left(D_{k} / D\right) T_{c 0}, \\
\Delta T_{h}^{A}=15.5-0.03\left(D_{k} / D\right) T_{c 0} .
\end{gathered}
$$

На рис. 4 кривые 1 и 2 иллюстрируют эти зависимости при $D_{k}=20 \mathrm{~nm}$ и $T_{c 0}=250 \mathrm{~K}$, пунктиром обозначена усредненная величина гистерезиса.

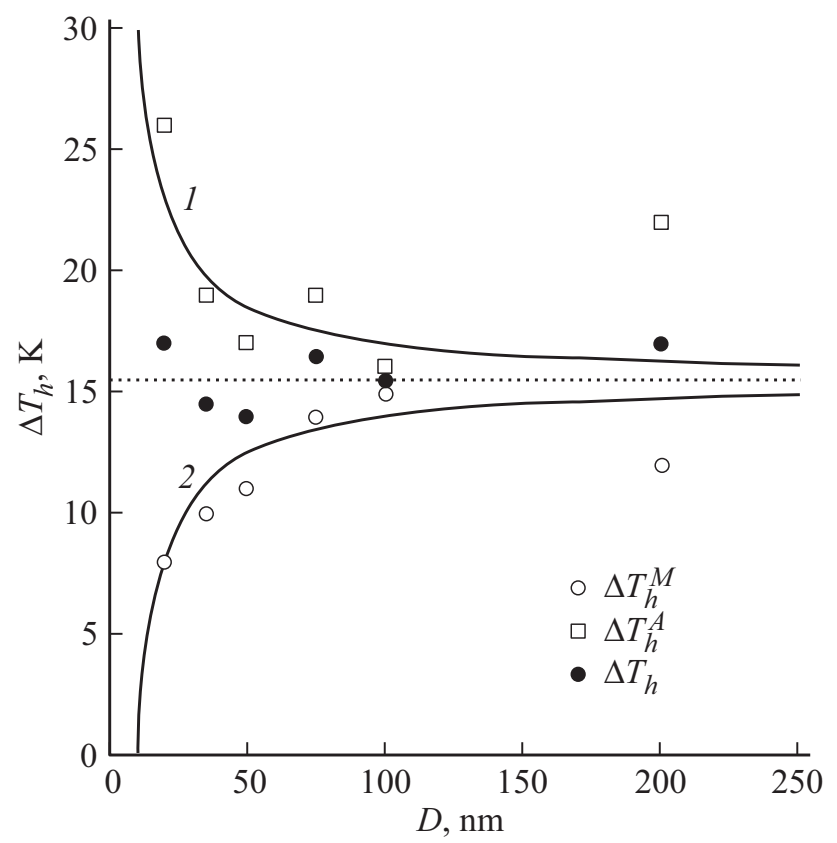

Рис. 4. Зависимость температурного гистерезиса превращения при прямом $\Delta T_{h}^{M}$ и обратном $\Delta T_{h}^{A}$ переходах и его средней величины $\Delta T_{h}$ в эпитаксиальных пленках сплава $\mathrm{Ni}_{51.6} \mathrm{Mn}_{32.9} \mathrm{Sn}_{15.5}$ от толщины пленок [2]. Кривые 1 и 2 - расчет согласно уравнениям (10), пунктир - средняя величина гистерезиса.

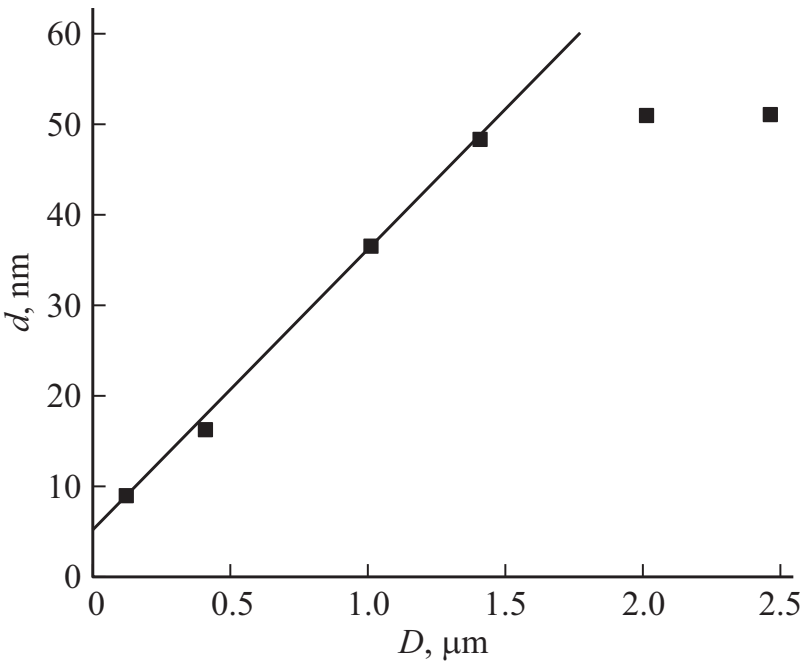

Рис. 5. Зависимость размера нанокристаллитов $d$ в эпитаксиальных пленках сплава $\mathrm{Ni}_{50} \mathrm{Mn}_{35} \mathrm{Sn}_{15}$ от толщины пленки $D$ [3]. Кривая - расчет согласно уравнению (11).

Приведенные в [3] калориметрические данные для пленок сплава $\mathrm{Ni}_{50} \mathrm{Mn}_{35} \mathrm{Sn}_{15}$, выращенных на кремниевых подложках, не так подробны, как данные [2]. Но достаточны для выяснения причины парадоксального расхождения между результатами [2] и [3] относительно критической толщины пленки $D_{k}$, меньше которой пленка остается в аустенитном состоянии. В [2] это $20 \mathrm{~nm}$, в [3] - критическая толщина равна $120 \mathrm{~nm}$. Согласно данным [3], пленки содержат нанокристаллиты размером $d$, величина которых увеличивается линейно с ростом толщины пленки $D$ вплоть до толщины $1.5 \mu \mathrm{m}$, когда в пленке начинается образование преципитатов, и она теряет когерентную связь с подложкой (рис. 5). На рис. 5 кривая иллюстрирует эмпирическую зависимость $d(D)$ на линейном участке,

$$
d(D)=5+D / 32.26 .
$$

Согласно уравнению (11) в поперечном сечении пленки имеется около 30 нанокристаллитов. Соотношение (11) позволяет с помощью приведенных в [3] данных найти зависимость упругих деформаций в пленке от размера нанокристаллитов $d$ (рис. $6, a$ и $b$ ). Кривые на этом рисунке построены согласно уравнению

$$
\varepsilon=\varepsilon_{0}+\frac{d_{0}}{32.26 d},
$$

где $d_{0}=1.5 \mathrm{~nm}, \varepsilon_{0}=0.45 \cdot 10^{-2}-$ деформация при экстраполяции $d \rightarrow \infty$ (рис. $6, b$ ). Видно, что величина упругих деформаций в пленке уменьшается с ростом размера кристаллов, но часть деформации $\varepsilon_{0}$ при $D>1.5 \mu \mathrm{m}$ не зависит от толщины пленки.

Нанокристаллизация пленки является результатом неоднородного распределения в ней упругих деформаций вследствие когерентной связи пленки с подложкой. В результате, в пленке возникают локальные 

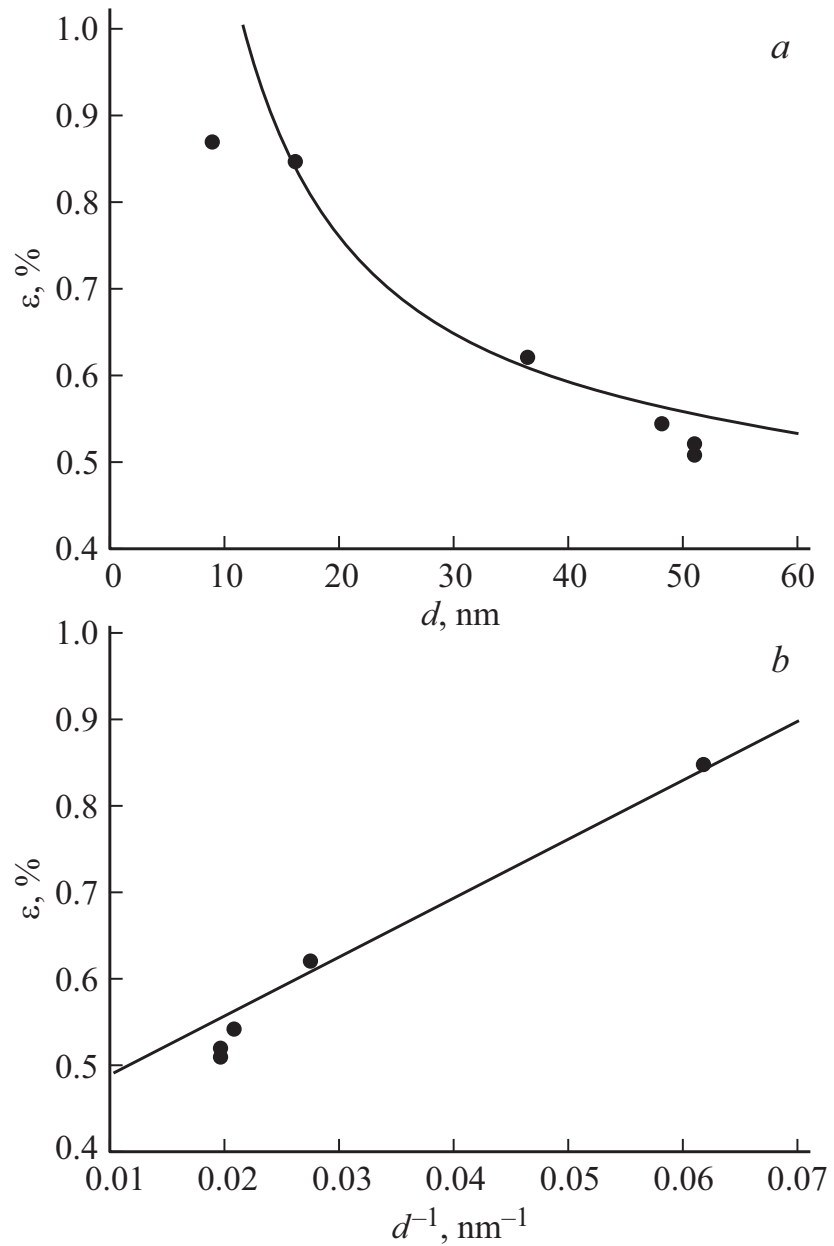

Рис. 6. Зависимость упругих микродеформаций в эпитаксиальных пленках сплава $\mathrm{Ni}_{50} \mathrm{Mn}_{35} \mathrm{Sn}_{15}$ от размера нанокристаллитов $d$ в пленке в прямых $(a)$ и обратных $(b)$ координатах [3]. Кривые - расчет по формуле (12).

напряжения из-за скоплений дислокаций одного знака на границах нанокристаллитов: ситуация аналогичная случаю когерентных с матрицей наночастиц $\mathrm{HfO}_{2}$ в сплаве $\mathrm{Cu}-\mathrm{Al}-\mathrm{Ni}[14]$. На языке дислокаций превращения релаксация упругих деформаций, связанных с нанозернами, происходит путем образования геометрически необходимых (ГН) дислокаций превращения. Согласно Эшби [17] такая ситуация имеет, например, место при наличии в кристалле неоднородных полей деформаций вокруг когерентных с решеткой частиц с поперечным размером $d$. При пластической деформации кристалла частицы становятся гетерогенными источниками образования решеточных ГН дислокаций. Применительно к дислокациям превращения формула Эшби для плотности дислокаций превращения в сплаве имеет вид

$$
\rho_{G}=\frac{\varepsilon_{m}}{2 a_{0} d}
$$

Среднее расстояние между дислокациями превращения $d_{G}$, т.е. есть диаметр дислокационной петли пре- вращения, определяющий элементарный объем превращения, равен $d_{G}=\sqrt{\rho_{G}}$. Для элементарного объема превращения получаем тогда следующее соотношение $\omega_{d}=\left(\pi d_{G}^{2} / 4\right) a_{0}=\pi a_{0} / 4 \rho_{G}$. Подставляя во вторую часть этого соотношения плотность ГН дислокаций (13), находим зависимость элементарного объема превращения в пленке от размера нанокристаллитов $d$,

$$
\omega_{d}=\frac{\pi a^{2}}{4 \varepsilon_{m}} d
$$

Сравним теперь величину объема $\omega_{d}$ и объема $\omega_{0}$ относительно друг друга, как это было сделано выше для сплава [2] (соотношения (5)). В результате находим, что $\omega_{0} / \omega_{d}=\varepsilon_{m}\left(D_{c}^{2} / a d \sqrt{2}\right) \approx 0.5-2.7$, при $\varepsilon_{m}=6 \cdot 10^{-2}, D_{c} \approx 14 \mathrm{~nm}$ и вариации $d$ в интервале 90-50 nm (рис. 5). Таким образом, в отличие от работы [2], стеснение (constrait) превращения вносит заметный вклад в зависимость ширины мартенситного перехода $\Delta T$ от размерного фактора $d$. К сожалению, в [3] нет данных относительно зависимости температурного интервала перехода от толщины пленки $D$ или от деформаций $\varepsilon$, что не позволяет, принимая во внимание соотношения (11), (12) и (14), установить зависимость ширины перехода от размера кристаллитов в пленке $\Delta T \sim 1 / \omega_{d}(d)$. В [3] указаны лишь две толщины пленки: $120 \mathrm{~nm}$, меньше которой она находится в аустенитном состоянии, и $400 \mathrm{~nm}$, выше которой - в мартенситном. Согласно соотношению (11) размер кристаллитов в этих пленках равен, соответственно, 8.7 и $17.4 \mathrm{~nm}$. Эти размеры того же порядка величины, что и критическая толщина пленки $D_{k}=20 \mathrm{~nm}$ наступления стабилизации аустенитного состояния в сплаве $\mathrm{Ni}-\mathrm{Mn}-\mathrm{Sn}$ [2], что устраняет кажущийся парадокс несоответствия результатов [2] и [3].

\section{2. Микрочастицы}

В микрочастицах порошка сплава $\mathrm{Ni}-\mathrm{Mn}-\mathrm{Sn}$ аналогичного по составу [3], полученных размолом сплава в шаровой мельнице и отожженных в течение 5 min при четырех температурах в интервале $573-873 \mathrm{~K}$, рентгеновская дифракция и мессбауерская спектроскопия обнаруживают наличие локальных деформаций наноразмерного масштаба [4,5]. Приведенные в [4] данные по зависимости величины деформаций и их пространственного размера от температуры отжига позволяют найти между ними прямую зависимость (рис. 7). Согласно этой зависимости по мере увеличения температуры отжига величина деформаций уменьшается, а их пространственный масштаб возрастает. Очевидно, что в микрочастицах сплава, пластически деформированного до высокой степени в шаровой мельнице, при отжиге развивается процесс нанокристаллизации. Наличие локальных упругих деформаций свидетельствует о когерентной связи нанокристаллов с решеткой сплава и наличии в границах кристаллитов скоплений дислокаций одного знака. На 
рис. 7 кривая показывает аппроксимацию найденных зависимостей уравнением

$$
\varepsilon(d)=-\varepsilon_{0}+\delta_{0} \frac{d_{k}}{d-d_{0}},
$$

где $\varepsilon_{0}=0.25 \cdot 10^{-2}$ - деформация при экстраполяции $d \rightarrow \infty, d_{0}=2 \mathrm{~nm}, \delta_{0}=0.94 \cdot 10^{-2}-$ деформация при $d=d_{k}=8.7 \mathrm{~nm}$. Видно, что локальные деформации в микрочастицах снижаются с ростом размера нанокристаллитов по такому же закону (12), что и в пленках [3].

В [4] приведена также зависимость температурного интервала мартенситного перехода $\Delta T$ от величины локальной деформации $\varepsilon$ в исходном после размола сплава состоянии и при четырех температурах отжига порошка. Эта зависимость имеет линейный характер. При экстраполяции ее к деформации $\varepsilon=0$ прямая пересекает ось ординат при $\Delta T=65 \mathrm{~K}$. Используя указанные на рис. 7 значения величины кристаллитов $d$ при различных температурах отжига, можно получить зависимость ширины мартенситного перехода $\Delta T$ от размера кристаллитов. Рис. 8 демонстрирует эту зависимость в координатах $\Delta T-1 / d$. Кривая на рисунке построена в предположении, что ширину мартенситного перехода определяют микронапряжения $\sigma_{e}=\mu \varepsilon(d)$ в микрочастицах сплава

$$
\Delta T(d)=\Delta T_{0}+\delta_{\sigma} \frac{d_{k}}{d} T_{c 0},
$$

где $\delta_{\sigma}=\delta_{0} \varepsilon_{m} \mu_{A} / q$. На рис. 8 кривая демонстрирует эту зависимость при $\delta_{\sigma}=0.14, d_{k}=8.7 \mathrm{~nm}, T_{c 0}=250 \mathrm{~K}$ и $\Delta T_{0}=57 \mathrm{~K}$ - результат экстраполяции кривой при

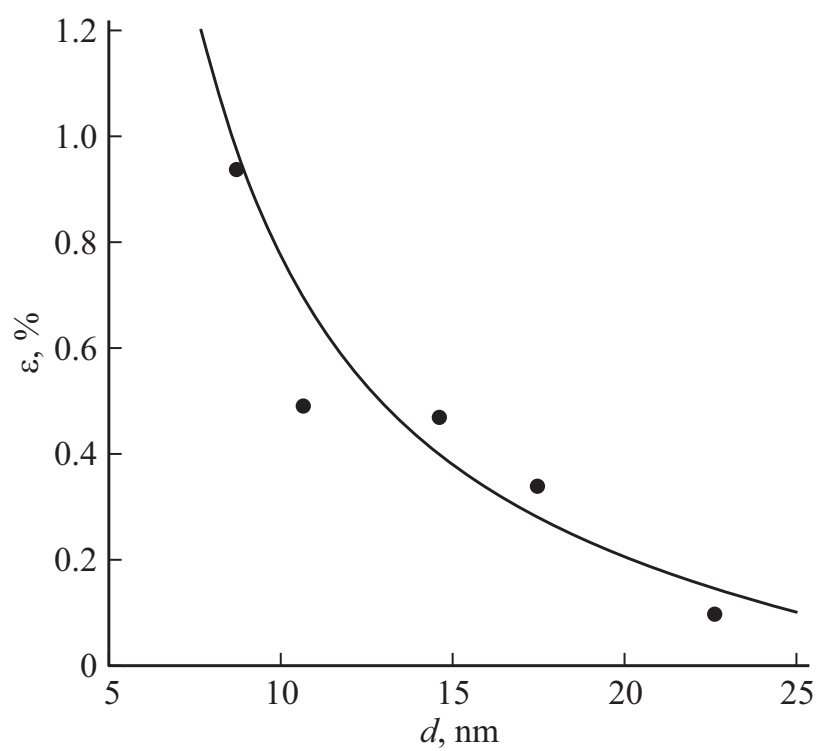

Рис. 7. Зависимость упругих микродеформаций $\varepsilon$ в отожженных в интервале температур 573-873 K микрочастицах порошка сплава $\mathrm{Ni}_{50} \mathrm{Mn}_{35} \mathrm{Sn}_{15}$ от размера нанокристаллитов $d$ в микрочастице $[4,5]$. Кривая - аппроксимация зависимости $\varepsilon(d)$ соотношением (15).

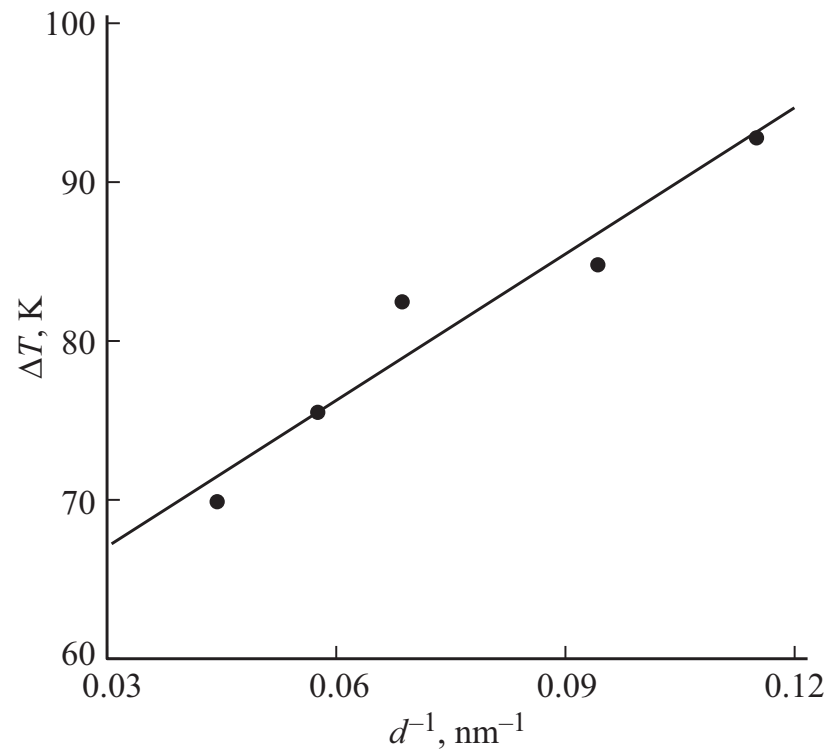

Рис. 8. Зависимость температурной ширины мартенситного превращения $\Delta T$ в микрочастицах порошка сплава $\mathrm{Ni}_{50} \mathrm{Mn}_{35} \mathrm{Sn}_{15}$ от величины нанокристаллитов $d$ в микрочастице [4]. Кривая - расчет согласно уравнению (16) в координа$\operatorname{таx} \Delta T(d)-1 / d$.

$d \rightarrow \infty$ до пересечения ее с осью ординат. Величина параметра $\delta_{\sigma}=0.14$ определяется вполне разумным набором данных $\delta_{0}=0.94 \cdot 10^{-2}, \varepsilon_{m}=6 \cdot 10^{-2}, \mu_{A}=7 \mathrm{GPa}$ и $q=30 \mathrm{MJ} / \mathrm{m}^{3}=30 \mathrm{MPa}$.

\section{4. Выводы}

Таким образом, сделанный в рамках теории РМП анализ размерных эффектов в эпитаксиальных пленках и микрочастицах сплава $\mathrm{Ni}-\mathrm{Mn}-\mathrm{Sn}$ позволяет сделать следующие выводы. Имеется три источником размерных эффектов в сплавах с ЭПФ.

1. Первый связан с существованием элементарного объема превращения $\omega$ вследствие пространственного ограничения (стеснения) фазового превращения. Было показано $[10,13]$, что при равновесной нанокристаллической структуре сплава или его монокристалльном наносостоянии элементарный объем превращения зависит от размера кристаллитов и поперечного размера кристалла, соответственно, как $\omega \sim d^{2}$ и $\sim D^{2}$, а температурный интервал перехода - как $\Delta T \sim 1 / \omega \sim 1 / d^{2}$ и $\sim 1 / D^{2}$.

2. Второй источник размерных эффектов - это наличие упругих напряжений и деформаций в эпитаксиальной пленке вследствие ее когерентной связи с подложкой, а также их наличие в микрочастицах сплава после его размола в шаровой мельнице. В монокристалическом и нанокристаллизованном состояниях пленки и микрочастиц эти напряжения вызывают снижение характеристических температур $T_{c}$ прямого и обратного мартенситных переходов и увеличивают температур- 
ную ширину перехода соответственно, как $\Delta T \sim 1 / D$ и $\sim 1 / d$.

3. Третий источник связан с кинетикой мартенситного перехода. Под кинетикой перехода в рамках теории РМП понимается не только изменение объемных долей фаз, но также их неоднородное распределение в кристалле сплава в виде ламелей (plates). В имеющейся литературе пока нет исследований по зависимости, например, ширины ламелей от поперечного размера кристалла или в нанокристаллическом материале - от величины зерен. Что касается, влияния размерного фактора на сам мартенситный переход, то выше экспериментально и теоретически продемонстрировано существование критической толщины пленки: в пленках меньшей толщины мартенситный переход блокируется.

\section{Список литературы}

[1] P. Czaja, R. Chulist, T. Tokarsky, T. Czeppe, Y.I. Chumlyakov, E. Cesari. J. Mater. Sci. 53, 10383 (2018).

[2] N. Teichert, A. Auge, I. Dincer, Y. Elerman, B. Krumme, H. Wednde, O. Yildirim, K. Potzger, A. Hütten. Acta Mater. 86, 279 (2015).

[3] R. Vishnoi, R. Singha, D. Kaur. J. Nanopart. Res. 13, 3975 (2011).

[4] J. López-García, I. Unzueta, V. Sánchez-Alarcos, V. Recarte, J.I. Pérez-Landzábal, J.F. Rodriguez-Velamazan, J.A. García, F. Plazaola. Intermetallics 94, 133 (2018).

[5] I. Unzueta, J. López-García, V. Sánchez-Alarcos, V. Recarte, J.I. Pérez-Landzábal, J.F. Rodriguez-Velamazan, J.S. Garitaonandia, J.A. García, F. Plazaola. Appl. Phys. Lett. 110, 181908 (2017).

[6] J.Y. Huang, Q.D. Hu, N.M. Bruno, J.H. Chen, I. Karaman, J.H. Ross, J.G. Li. Scr. Mater. 105, 42 (2015).

[7] V. Sá nchez-Alarcos, J.I. Pérez-Landzábal, V. Recarte, I. Lucia, J. Vélez, J.A. Rodríguez-Velamezán. Acta Mater. 61, 4676 (2013).

[8] P.O. Castillo-Villa, L. Manosa, P. Planes, D.E. Soto-Parra J.L. Sanches-Llamazares, H. Flores-Zuniga, C. Frontera. J. Appl. Phys. 113, 053506 (2013).

[9] Г.А. Малыгин. УФН 171, 187 (2001).

[10] Г.А. Малыгин. ФТТ 61, 288 (2019).

[11] W.S. Ko, S.B. Maisel, B. Grabowski, J.B. Leon, J. Neugebauer. Acta Mater. 123, 90 (2017).

[12] N. Ozdemir, I. Karaman, N.F. Mara, Y.I. Chumlyakov, H.E. Karasa. Acta Mater. 60, 5670 (2012).

[13] Г.А. Малыгин. ФТТ 60, 1990 (2018).

[14] Г.А. Малыгин, В.И. Николаев, С.А. Пульнев. ЖТФ 89, 1 , 132 (2019).

[15] H. Zong, Z. Ni, X. Ding, N. Lookman, J. Sun. Acta Mater. 103, 407 (2016).

[16] Z. Chen, S. Qin, J. Shang, Y. Chen. Intermetallics 94, 47 (2018).

[17] A.F. Ashby. Phil. Mag. 21, 399 (1970).

Редактор Т.Н. Василевская 